\title{
Metodologia INTERA para o desenvolvimento de Objetos de Aprendizagem
}

\author{
Juliana Cristina Braga, Edson Pimentel, Silvia Dotta
}

Centro de Matemática, Computação e Cognição - Universidade Federal do ABC (UFABC) - Grupo de pesquisa INTERA

\{juliana.braga,edson.pimentel, silvia.dotta\}@ufabc.edu.br

\begin{abstract}
The production of Learning Objects $(L O)$ is very complex because it involves contributions of the multidisciplinary team with different skills. These professionals need together reach the objectives technological and pedagogical of these products. In this directions, it is mandatory the use of methodologies to organize the processes of development, standardization and communication among stakeholders. The use of an inadequate methodology could be result ineffective $L O$ and its reuse in the learning as it is likely to be provided. The methods used for the development of $L O$ also have gaps at both question as well as technical teaching in some respects as evidenced therein. In short, there is a lack of methodologies for the production of LO that take into account the characteristics of software development cycles, treatment needs teaching and pedagogical. Marking to fill this gap, this paper presents the methodology INTERA for the development of learning objects.
\end{abstract}

Resumo. A produção de um Objeto de Aprendizagem (OAs) é bastante complexa, pois envolve a participação de uma equipe multidisciplinar, composta por professores, desenvolvedores, designers gráficos e especialistas de área computacional. Esses profissionais devem interagir de modo a atingir os objetivos tanto tecnológicos quanto pedagógicos desses produtos. Nesse sentido, torna-se necessário o uso de metodologias para organizar o processo de desenvolvimento, a padronização e a comunicação entre os envolvidos. $O$ uso de uma metodologia inadequada pode resultar em $O A$ ineficazes em seu reuso e no aprendizado que ele possa vir a fornecer. As metodologias que vem sendo utilizadas para o desenvolvimento de $O A$ apresentam falhas tanto na questão técnicas como também em alguns aspectos pedagógicos. Visando preencher nessa lacuna, esse artigo apresenta a metodologia INTERA para o desenvolvimento de objetos de aprendizagem.

\section{Introdução}

Segundo pesquisa recente realizada pelo Centro de Estudos sobre as Tecnologias da Informação e da Comunicação (CETIC), o uso das tecnologias no âmbito educacional tem crescido a cada ano no Brasil (CETICbr, 2012). Nesse contexto, os materiais instrucionais digitais utilizados para o ensino têm sido classificados como Objetos de Aprendizagem (OAs) e podem auxiliar o professor a criar novas estratégias pedagógicas que favoreçam o aprendizado do aluno. Apesar de ser um desafio, espera-se que um mesmo OA possa ser reutilizado em diferentes contextos educacionais (Braga et al., 2012). 
Por ser tratar de um conteúdo digital voltado ao aprendizado, um OA deve conter tanto qualidade técnica como pedagógica, caso contrário, sua utilização pode acarretar desmotivação do aluno e, no pior caso, conduzir a um aprendizado inadequado.

A necessidade de se produzir OAs de qualidade impõe uma reflexão sobre o papel do professor para além de planejar, preparar e conduzir o conteúdo de uma aula, o novo papel docente é sua atuação no processo de produção de conteúdos instrucionais digitais mais complexos.

O processo de produção de OAs deve contar com a participação dos professores, no entanto, afora dominar sua área de atuação, são poucos os professores que possuem conhecimentos técnicos suficientes para a produção de OAs com alta qualidade e reuso. Apesar dessas dificuldades técnicas, a participação dos professores nesse processo é fundamental, já que eles são os detentores dos componentes pedagógicos desse procedimento. Em sentido contrário, nem sempre os professores são detentores do conhecimento técnico que envolve o desenvolvimento de OAs, necessitando assim do apoio de equipes multidisciplinares para sua produção. Essas equipes variam conforme o tipo de OA (cursos, vídeos, simulações, softwares, imagens, etc), mas podem ser compostas por cientistas da computação, videomakers, designers, músicos etc.

Notoriamente o desenvolvimento de OAs é uma área interdisciplinar e não pode ser considerado um processo trivial. Sendo assim, torna-se importante o uso de metodologias apropriadas para organizar, padronizar, facilitar a comunicação entre os envolvidos nesse processo. $\mathrm{O}$ uso de metodologias inadequadas ou a não adoção de uma metodologia pode gerar OAs que não atendam as necessidades de aprendizagem dos alunos e/ou OAs de baixa qualidade técnica.

Existem algumas metodologias que estão sendo utilizadas na produção de OAs, algumas delas são baseadas somente em abordagens para elaboração de conteúdos instrucionais com ênfase na parte pedagógica, outras baseadas em processos de desenvolvimento de software com ênfase na parte técnica. Entretanto, conforme a ser discutido nesse trabalho, é necessário criar novas tecnologias que encontrem o equilíbrio entre a área técnica e a área pedagógica.

Diante da importância dessas metodologias e das deficiências que as metodologias atuais apresentam para o desenvolvimento de OAs, este artigo possui como objetivo propor uma metodologia mais adequada para esse campo. Para tanto, partimos da análise das principais metodologias para desenvolvimento de OAs, destacando suas vantagens e deficiências, a partir de uma perspectiva do design instrucional.

\section{Metodologias e Processos para o Desenvolvimento de Objetos e Aprendizagem}

Existem três tipos de abordagens que vem sendo utilizadas para o desenvolvimento de OAs: a primeira são as metodologias que consideram fortemente o design instrucional (os aspectos pedagógicos), outras que consideram somente $\mathrm{o}$ processo de desenvolvimentos de softwares (aspectos computacionais) e outras que mesclam um pouco desses dois aspectos. Essa seção apresenta algumas dessas metodologias e suas 
deficiências no âmbito pedagógico.

\subsection{Design Instrucional (DI): abordagem ADDIE}

Design Instrucional (DI) é definido como um processo sistemático utilizado para desenvolver cursos de educação e formação de uma forma consistente e confiável (livre de falhas) (Branch, 2009). O DI torna-se cada vez mais necessário no contexto da educação on-line, sendo desenvolvido por várias instituições, que buscam uma melhor interação entre o usuário e o sistema.

De acordo com Filatro (2004), design instrucional é a ação institucional e sistemática de ensino, que envolve o planejamento, o desenvolvimento e a utilização de métodos, técnicas, atividades, materiais, eventos e produtos educacionais em situações didáticas específicas, a fim de facilitar a aprendizagem humana a partir dos princípios de aprendizagem e instrução conhecidos.

Uma das abordagens mais antigas e utilizadas para o DI é a metodologia ADDIE, acrônimo em inglês de Analyze (Analisar), Design (Projetar), Develop (Desenvolver), Implement (Implementar), and Evaluate (Avaliar). ADDIE é um paradigma de desenvolvimento de produtos em geral, mas tem sido muito aplicado para um tipo específico de produto que são os materiais instrucionais, no entanto possui baixa ênfase nos aspectos computacionais de um OA, uma vez que quando a metodologia ADDIE foi criada, o conceito de OA ainda não existia.

\subsection{Processo RIVED (Rede Interativa Virtual de Educação)}

O RIVED (RIVED, 2008) foi um excelente programa da Secretaria de Educação a Distância (SEED), que teve por objetivo a produção de conteúdos pedagógicos digitais, na forma de OAs baseados em simulações e animações.

O modelo de processo de produção de OAs proposto pelo RIVED é baseado na junção de um conjunto de características que são pertinentes ao aprendizado do indivíduo. Essa junção dá origem aos documentos gerados durante o processo: a) General Design (GD) ou Design Pedagógico,b) Roteiro de Atividades e c) Guia do Professor. Todos eles são gerado nas 6 fases desse processo. Apesar de ser um modelo específico para OAs, o mesmo foi elaborado e validado somente para OAs do tipo animação e simulação.

Esse modelo possui deficiências quando aplicado a outros tipos de objetos como: softwares, cursos a distâncias ou vídeos.

\subsection{Processo SOPHIA}

O processo SOPHIA (Pessoa, 2008) foi proposto pela equipe do Laboratório de Soluções em Software da Universidade do Vale do Itajaí (UNIVALI). Segundo os autores, ele não é apenas um processo, mas um ambiente (repositório) que agrupa OAs.

O processo SOPHIA de produção de OAs é composto por três etapas distintas: projeto, desenvolvimento e distribuição. Apesar de ser um processo criado especificamente para a produção de OAs, possui baixa ênfase em questões pedagógicas, como por exemplo, a avaliação pedagógica. 


\subsection{RUP}

Os processos de desenvolvimento de softwares têm sido bastante utilizados para o desenvolvimento de OAs. No entanto, nessa seção escolheu-se apresentar somente o processo Rational Unified Process (RUP) (Kruchten, 2003), por ser considerado um processo mais completo, mesmo que muitas vezes considerado não ágil. $\mathrm{O}$ fato de ser mais completo permite uma análise que pode ser estendido para outros processos mais ágeis, porem menos completos.

Diferentemente dos outros processos mencionados nesse artigo (RIVED, SOPHIA e ADDIE) o RUP não possui etapas, mas sim disciplinas. Uma disciplina é uma coleção de tarefas que estão relacionadas a uma área de interesse principal dentro do projeto como um todo. Por ter sido criado para o desenvolvimento de softwares em geral, o RUP é um processo bem completo, mas não aborda em nenhuma de suas etapas a questão pedagógica.

\subsection{Comparações das metodologias sob a perspectiva pedagógica}

Nesta seção, as metodologias ADDIE, RIVED, SOPHIA e RUP são comparadas sob a perspectiva do design instrucional.

Para direcionar esse comparativo foi realizado um estudo bibliográfico das metodologias em questão no qual foram identificadas as principais atividades pedagógicas encontradas em cada uma delas. Todas as atividades identificadas são apresentadas na primeira coluna da tabela 1, sendo que na segunda coluna é indicado em qual das metodologias cada atividade foi encontrada.

Tabela 1 - Identificação das atividades de design instrucional nas metodologias utilizadas para o desenvolvimento de objetos de aprendizagem.

\begin{tabular}{|l|l|}
\hline \multicolumn{1}{|c|}{ Atividades do DI } & \multicolumn{1}{|c|}{ Metodologia que contém a atividade } \\
\hline Analisar as necessidades de aprendizagem & ADDIE, RIVED \\
\hline Identificar o problema de aprendizagem & ADDIE \\
\hline Concepção de um currículo ou programa & ADDIE, RIVED \\
\hline $\begin{array}{l}\text { Selecionar e utilizar técnicas para determinar o conteúdo } \\
\text { instrucional }\end{array}$ & ADDIE, RIVED \\
\hline $\begin{array}{l}\text { Analisar as características do Ambiente Virtual de } \\
\text { Educação }\end{array}$ & ADDIE \\
\hline $\begin{array}{l}\text { Analisar as características das tecnologias para uso no } \\
\text { ambiente de ensino }\end{array}$ & ADDIE \\
\hline Estabelecer vocabulário comum & ADDIE, RUP \\
\hline Planejar a situação didática & ADDIE, RIVED, SOPHIA \\
\hline Mapeamento e seqüenciamento do conteúdo & ADDIE, RIVED, SOPHIA \\
\hline Definir estratégias e atividades & ADDIE \\
\hline $\begin{array}{l}\text { Preparação dos suportes (pedagógicos, tecnológicos e } \\
\text { administrativos) }\end{array}$ & ADDIE, RIVED, SOPHIA \\
\hline Desenvolver medidas de avaliação & ADDIE \\
\hline $\begin{array}{l}\text { Avaliar produtos resultantes de cada fase do DI (relatório } \\
\text { de analise, storyboards ou roteiros, interface do curso ou } \\
\text { material instrucional, relatórios de acompanhamento, } \\
\text { relatórios finais de avaliação }\end{array}$ & ADDIE \\
\hline Avaliar solução Educacional & ADDIE \\
\hline Realizar plano de avaliação formativa/somativa & ADDIE \\
\hline
\end{tabular}


A Análise da tabela 1 leva as seguintes conclusões: i) a abordagem ADDIE contém todas as atividades de Design Instrucional identificadas no estudo; ii) o modelo RIVED contém apenas seis das quinze atividades de DI; iii) o processo SOPHIA possui apenas três atividades pedagógicas; iv) o processo RUP aborda somente uma atividade pedagógica.

Complementando esse estudo com outra análise, realizada pelo mesmo grupo, porém sobre a perspectiva de reuso e qualidade técnica de OAs (Braga, et al. 2012), pode-se concluir que: i) abordagem ADDIE é a que possui maior enfoque pedagógico, mas por outro lado possui grade deficiência na qualidade técnica e no reuso. ii) o RUP é o que possui maior deficiência na parte instrucional, mas é considerado o processo mais completo tecnicamente, pois aborda questões sobre qualidade e reuso de objetos. iii) $\mathrm{O}$ SOPHIA e o RIVED é mais adequado que o ADDIE em termos técnico e de reuso e menos adequado que o RUP nessas mesmas questões.

Partindo da análise realizada nessa seção e da análise de estudos anteriores (Braga, et al) conclui-se que uma metodologia adequada seria aquela que contemplasse as atividades pedagógicas contida na abordagem ADDIE e as atividades computacionais contidas nas disciplinas do RUP. Foi seguindo essa linha de raciocínio que esse artigo propõe uma nova metodologia denominada INTERA. Essa metodologia foca no reuso, qualidade técnica e também no Design instrucional.

\section{Proposta da Metodologia INTERA}

A Metodologia INTERA (Inteligência, Tecnologias Educacionais e Recursos Acessíveis) foi inspirada em processos de desenvolvimento de software e no modelo ADDIE para desenvolvimento de conteúdos instrucionais.

A metodologia proposta deve ser considerada um arcabouço de processos para o desenvolvimento de qualquer tipo de conteúdo digital utilizado para a aprendizagem. Isso implica que é aderente a qualquer tipo de OAs, independente de sua granularidade, seu tamanho ou complexidade.

A metodologia INTERA é uma metodologia iterativa, que sugere o "ir e vir" em suas etapas. A metodologia INTERA considera o processo de desenvolvimento de OAs como sendo um projeto e por isso ela também contempla que o ciclo de vida de um OA define as fases que conectam o início de um projeto de desenvolvimento de OA ao final. Essa consideração é baseada no guia do conjunto de conhecimentos em gerenciamento de Projetos (PMBOK, 2008), um dos pilares no qual a metodologia INTERA se apoia.

Independente do tipo do OA, a metodologia INTERA possui os seguintes componentes: fases, papeis, etapas e artefatos. A seguir um detalhamento sobre esses componentes:

Fases: são os períodos que as etapas da metodologia podem pertencer, sendo que uma etapa pode pertencer a mais de uma fase. As fases geralmente são sequenciais e normalmente são definidas pela entrega de algum componente do OA. São três as fases da metodologia INTERA: inicial, intermediária e transição.

Papeis: não são pessoas, pelo contrário, eles descrevem como as pessoas devem se comportar no projeto e quais são suas funções. Sendo assim, uma pessoa pode 
assumir mais de um papel na metodologia.

A definição do papel dos envolvidos logo no início do projeto do OA é extremamente importante, pois entendemos que é crucial a compreensão inicial das responsabilidades de cada um, já que o sucesso na comunicação entre os pares, na compreensão das suas atribuições e do processo é o que acarretará no sucesso do desenvolvimento do OA. A definição de papeis da metodologia INTERA foi baseada no processo unificado de engenharia de software (RUP), no modelo de design instrucional ADDIE e no processo RIVED.

São considerados papeis na metodologia INTERA: analista, conteudista, gerente de projetos, demandante, designer de interface, designer instrucional, equipe de desenvolvimento e Equipe de Teste. Cada um dos papeis são descritos na tabela 2.

Tabela 2 - Descrição dos papeis da metodologia INTERA.

\begin{tabular}{|c|c|}
\hline Papel & Descrição das atividades do papel \\
\hline Analista & $\begin{array}{l}\text { Responsável em fazer o levantamento e análise do contexto e dos requisitos do OA. } \\
\text { Também é responsável por elaborar o planejamento da qualidade e dos testes do OA. }\end{array}$ \\
\hline Conteudista & $\begin{array}{l}\text { Responsável pela elaboração do conteúdo, incluindo pesquisa de conteúdo, } \\
\text { especificação de conteúdos adicionais e avaliação do conteúdo na etapa de testes. É } \\
\text { também sua função por manter a integridade do conteúdo do OA realizando várias } \\
\text { revisões no mesmo, se necessário. Deverá manter o OA dentro dos objetivos } \\
\text { pedagógicos no qual ele foi concebido e garantir a qualidade e veracidade do } \\
\text { conteúdo. }\end{array}$ \\
\hline Demandante & $\begin{array}{l}\text { Solicita o desenvolvimento do OA que será desenvolvido. Pode ser o principal } \\
\text { responsável por fornecer a verba necessária para o desenvolvimento do OA. }\end{array}$ \\
\hline Gerente de Projetos & $\begin{array}{l}\text { Responsável por planejar e gerenciar o projeto de desenvolvimento do OA. Faz parte } \\
\text { de suas atribuições: manter a comunicação entre a equipe, acompanhar o cronograma, } \\
\text { escopo e custo do projeto, distribuir e gerenciar as atividades da equipe. }\end{array}$ \\
\hline Designer de Interface & $\begin{array}{l}\text { Projeta os componentes de interface do OA de forma a potencializar o entendimento } \\
\text { do conteúdo (produzido pelo conteudista) a partir do uso de linguagens e formatos } \\
\text { variados (hipertexto, da mixagem e da multimídia). Desenvolve a identidade visual do } \\
\text { objeto. }\end{array}$ \\
\hline $\begin{array}{l}\text { Designer Instrucional } \\
\text { (designer pedagógico) }\end{array}$ & $\begin{array}{l}\text { Será o responsável por realizar o planejamento pedagógico a avaliação pedagógica do } \\
\text { OA. }\end{array}$ \\
\hline $\begin{array}{l}\text { Designer Técnico (ou } \\
\text { Arquiteto) }\end{array}$ & $\begin{array}{l}\text { Responsável pelas escolhas tecnológicas para o desenvolvimento do OA, de acordo } \\
\text { com seu contexto e requisitos. Também responsável por fornecer subsídios técnicos } \\
\text { de forma a guiar a equipe de desenvolvimento. Exerce a função de um consultor } \\
\text { técnico e necessita ter formação na área de computação ou similar. }\end{array}$ \\
\hline $\begin{array}{l}\text { Equipe de } \\
\text { desenvolvimento }\end{array}$ & $\begin{array}{l}\text { Responsável pelo desenvolvimento ou produção do OA. Essa equipe deverá ser } \\
\text { formada por profissionais técnicos de acordo com o tipo de OA a ser desenvolvido. } \\
\text { Exemplos: se o curso precisar de um vídeo, a equipe deverá possuir técnicos em } \\
\text { produção de vídeo. Se a equipe precisar de um software, a equipe deverá constituir de } \\
\text { programadores. }\end{array}$ \\
\hline Equipe de teste & $\begin{array}{l}\text { Responsável por realizar diferentes tipos de testes ao longo do desenvolvimento do } \\
\text { curso garantindo assim sua qualidade. Faz parte de suas atribuições testar as } \\
\text { funcionalidades, a acessibilidade, confiabilidade, etc. }\end{array}$ \\
\hline
\end{tabular}

Artefatos: em quase todas as etapas da metodologia, dados são gerados pela equipe envolvida, desde documentos até código fonte (caso o OA seja um software). Todos esses dados, contendo informações, são considerados artefatos para a metodologia INTERA.

Etapas: são coleções de atividades relacionadas a uma área de interesse 
principal. A duração de uma etapa depende do tipo de objeto e de sua complexidade. As etapas podem ocorrer concomitantemente ou não, variam de acordo com o modelo de desenvolvimento adotado pela equipe.

Dentro de cada etapa deve existir um conjunto de atividades e práticas que variam conforme o tipo de objeto. A definição dos nomes das etapas da metodologia INTERA foi baseada no "Guide to the Software Engineering Bodyof Knowledge" (SWEBOK, 2004), no entanto o referido guia considera área de conhecimento o que consideramos como etapa na metodologia. O grupo considera que o nome "etapas" é melhor compreensível para os profissionais das áreas menos técnicas e envolvidos na produção de OAs.

As etapas da metodologia INTERA são: contextualização, requisitos, design (ou arquitetura), desenvolvimento, testes e qualidade, disponibilização, avaliação, gestão de projetos e ambiente e padrões. Essas etapas são interativas, sendo integradas pela gestão de projetos conforme a Figura 1a.

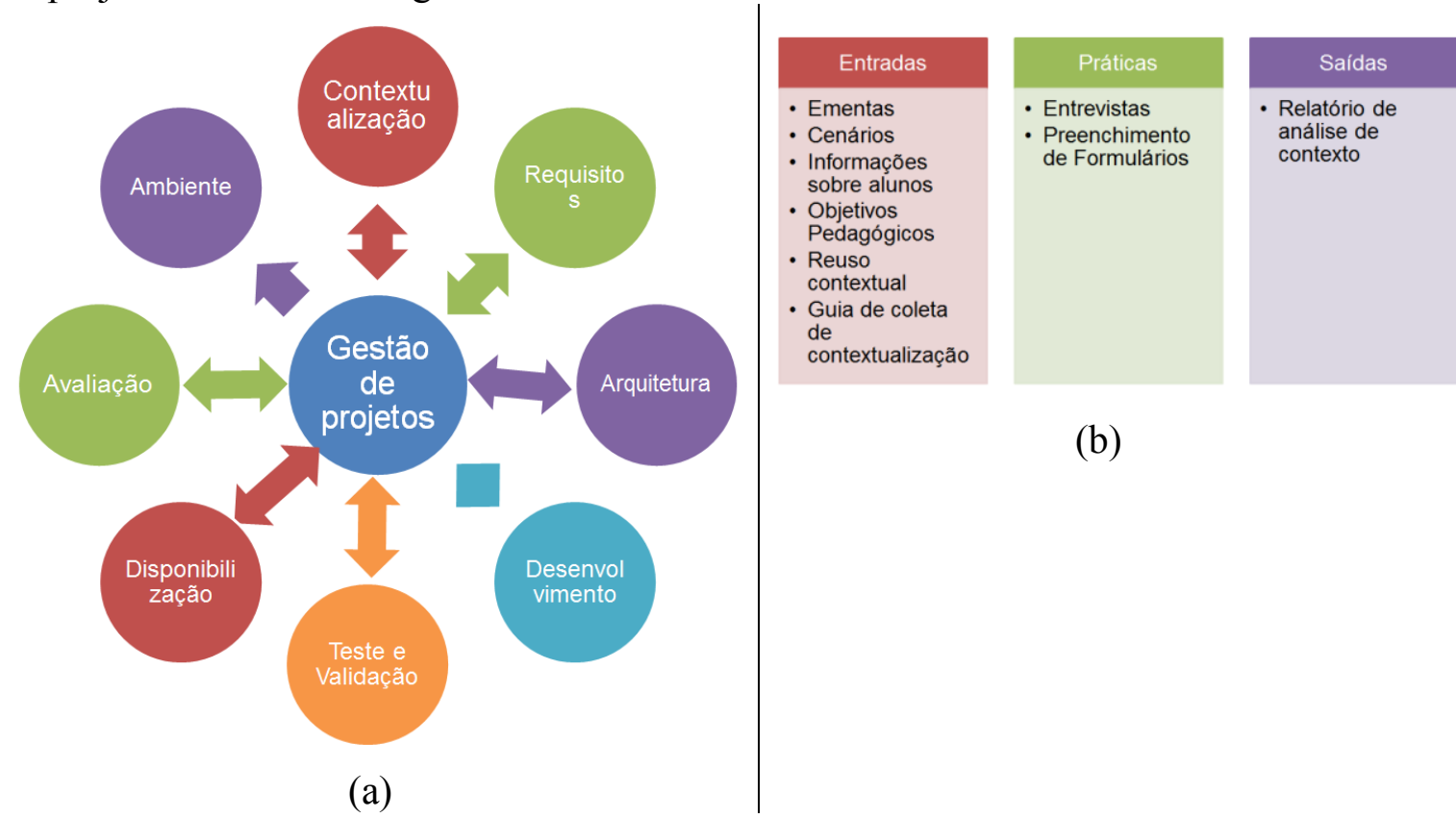

Figura 1 - (a) Etapas da metodologia INTERA. (b) Etapa de Contextualização com seus elementos de entrada, práticas e elementos de saída (exemplo em um curso on-line).

Cada etapa é divindade em 3 elementos: i) entrada; ii) práticas e iii) saída. Os elementos_de entrada são as informações e/ou artefatos necessários para $\mathrm{o}$ desenvolvimento das etapas. As práticas são técnicas já consagradas e utilizadas na área de computação e/ou educação que podem ser usadas no desenvolvimento de um AO e variam conforme o seu tipo. Como exemplos de práticas pode-se citar: storyboard, roteiros de vídeos, protótipos de softwares, sumário executivo de cursos. Essas práticas não fazem parte da metodologia INTERA, no entanto a metodologia recomenda algumas delas. Outros elementos e técnicas também podem ser introduzidos na metodologia de acordo com a necessidade da equipe de trabalho. Os elementos de saída são informações ou artefatos gerados durante ou no final do desenvolvimento da etapa. Figura $1 \mathrm{~b}$ mostra os três elementos somente para a etapa de contextualização, o mesmo 
poderia ser feito para as outras etapas.

A seguir um detalhamento de cada etapa da metodologia.

Contextualização: definição do contexto pedagógico OA deverá ser aplicada. Recomenda-se que essa seja a primeira etapa a ser iniciada. Pois é necessário conhecer bem em que condições e qual o público alvo do OA. Exemplos de informações dessa etapa: ementa em que o curso se encaixa, descrição do público alvo, modalidade de ensino, objetivo de aprendizagem, possibilidade de ser utilizado por pessoa portadora de deficiência (acessibilidade), cenário e contexto em que ele se apresentará etc. $\mathrm{O}$ principal artefato dessa etapa é o documento contextualização.

Requisitos: levantamento do que se espera do OA, suas características técnicas e pedagógicas. Nesta etapa, são gerados os principais artefatos e o professor deve atuar fortemente nesse momento.

Design ou Arquitetura: envolve a análise dos requisitos que resultará no esboço (ou rascunho) do OA. Nesta etapa, também são definidas as tecnologias mais adequadas para o desenvolvimento do OA e os padrões a serem adotados. Também nessa etapa são definidos os componentes de reuso.

Desenvolvimento: é nessa etapa que o objeto de aprendizagem e todo o seus componentes de reuso (manual do usuário, instalação, guia de edição, etc) são desenvolvidos.

Testes e Qualidade: realização de validações das características técnicas (incluindo acessibilidade e usabilidade) e parte das características pedagógicas levantadas nas etapas anteriores. Verificação da qualidade também é uma atividade importante dessa etapa, pois entendemos que isso está intimamente ligado aos testes.

Disponibilização: etapa em que é realizada a disponibilização (ou publicação) do objeto, da documentação de uso e instalação em repositórios.

Avaliação: é a aplicação do objeto em sala de aula, com o objetivo principal de avaliar seu aprendizado.

Gestão de projetos: Esta etapa perpassa por todo o processo e representa a execução das funções do coordenador acompanhando e analisando os custos, cronograma e os envolvidos.

Ambiente: Esta etapa é responsável por controlar o ambiente técnico em que o OA está sendo desenvolvido, como por exemplo, a realização de backups e controle de versionamento.

Em linha com o objetivo desse artigo, para realizar a conexão entre as etapas da metodologia INTERA e evidenciar que essa metodologia, assim como a abordagem ADDIE, apresenta todas as atividades pedagógicas identificadas no estudo relatado na seção 2.2, deve-se observar a tabela 3. Nessa tabela, cada atividade apresentada na coluna 1 pertence a uma etapa da metodologia INTERA descrita nessa seção e identificada na coluna 2 . 
Tabela 3 - Atividades pedagógicas apresentada na metodologia INTERA.

\begin{tabular}{|l|l|}
\hline \multicolumn{1}{|c|}{ Atividades do DI } & \multicolumn{1}{|c|}{ Etapa da metodologia INTERA } \\
\hline Analisar as necessidades de aprendizagem & Contextualização \\
\hline Identificar o problema de aprendizagem & Contextualização \\
\hline Concepção de um currículo ou programa & Requisitos \\
\hline $\begin{array}{l}\text { Selecionar e utilizar técnicas para determinar or o } \\
\text { conteúdo instrucional }\end{array}$ & Requisitos \\
\hline $\begin{array}{l}\text { Analisar as características do Ambiente Virtual de } \\
\text { Educação }\end{array}$ & Desin. \\
\hline $\begin{array}{l}\text { Analisar as características das tecnologias para uso no } \\
\text { ambiente de ensino }\end{array}$ & Design \\
\hline Estabelecer vocabulário comum & Contextualização \\
\hline Planejar a situação didática & Design \\
\hline Mapeamento e sequenciamento do conteúdo & Design \\
\hline Definir estratégias e atividades & Design \\
\hline $\begin{array}{l}\text { Preparação dos suportes (pedagógicos, tecnológicos e } \\
\text { administrativos) }\end{array}$ & Ambiente \\
\hline Desenvolver medidas de avaliação & Avaliação \\
\hline Preparar plano de Avaliação & Avaliação \\
\hline $\begin{array}{l}\text { Avaliar produtos resultantes de cada fase do DI } \\
\text { (relatório de analise, storyboards ou roteiros, interface } \\
\text { do curso ou material instrucional, relatórios de } \\
\text { acompanhamento, relatórios finais de avaliação }\end{array}$ & Avaliação \\
\hline Realizar plano de avaliação formativa/somativa & Avaliação \\
\hline
\end{tabular}

\section{Considerações finais}

A produção de OAs é bastante complexa, pois envolve a participação de uma equipe multidisciplinar. Esses profissionais devem interagir de modo a atingir os objetivos tanto tecnológicos quanto pedagógicos desses produtos. Nesse sentido, torna-se necessário o uso de metodologias para evitar a criação de OA ineficazes em seu reuso e no aprendizado que ele possa vir a fornecer.

Esse artigo discute que existe uma carência de metodologias de produção de OA que levem em consideração ao mesmo tempo as características técnicas de um OA com as necessidades de tratamento didático-pedagógicas.

Visando preencher essa lacuna, a metodologia INTERA considera em suas etapas todas as atividades pedagógicas que são baseadas em metodologias de design instrucional e que fizeram parte do estudo apresentado nesse artigo. A partir desse estudo, pode-se concluir que a metodologia INTERA aborda de forma adequada a questão pedagógica que é considerada inadequada em outros processos. Por outro lado, os estudos anteriores comprovam que, dentre as metodologias estudadas, a metodologia INTERA também é a mais adequada para questões técnica e de reuso de OAs.

Ressalta-se a preocupação do grupo em não somente fundamentar a metodologia, mas também validá-la no desenvolvimento de diferentes tipos de objetos de aprendizagem. Isso tem sido feito, até o momento, para OAs do tipo curso a distância (Dotta, et al), do tipo aula virtual (Braga, 2013), do tipo software educacional (Siller, 2013) e do tipo animação. Para todos os tipos de OAs as etapas são as mesmas, variando os elementos de entrada, os de saída e as práticas ou técnicas selecionadas. Para todas as validações a metodologia tem se mostrado adequada ao que se propõe que 
é guiar o desenvolvimento de objetos de aprendizagem com foco dos aspectos pedagógicos e nas características de reuso. Além disso, a metodologia favorece a comunicação entre a equipe interdisciplinar.

Acredita-se ser importante para o uso efetivo da metodologia o seu treinamento e divulgação. Nessa linha, cerca de 10 professores universitários foram treinados na metodologia INTERA, esses professores têm relatado que, após o treinamento, eles passam a perceber a complexidade no processo de produção de um OA de qualidade e a importância de seu papel nesse processo. Outros profissionais também já foram treinados, totalizando aproximadamente 30 pessoas. Esses treinamentos têm sido importantes também como forma de validação da metodologia, onde as dificuldades são evidenciadas e a metodologia ajustada em função desses relatos.

\section{Referências}

Braga, J. C. ; Bandeira, R. C. ; Marcondes, R. R. ; Dotta, S. ; Pimentel, E. (20013). Validando a metodologia INTERA no desenvolvimento de um Objeto de aprendizagem do tipo aula virtual. In: X Congresso Brasileiro de Ensino Superior a Distância - ESUD 2013, 2013, Belém. Anais do X Congresso Brasileiro de Ensino Superior a Distância - ESUD 2013.

Braga, J. C. (2009). "Diretrizes para o Ensino Interdisciplinar de Engenharia de Software". Anais do FEES09 - Fórum de Educação em Engenharia de Software, Fortaleza, Outubro.

Braga, J. C., Pimentel, E., Dotta, S. (2012) Desafios para o Desenvolvimento de Objetos de Aprendizagem Reutilizáveis e de Qualidade. Anais do DesafIE!/2012 - Workshop de Desafios da Computação Aplicada à Educação, 17 e 18 de Julho de 2012 - Curitiba/PR - CEIE/SBC.

Branch R. M (2009) "Instructional Design: The ADDIE Approach.” Springer Science+Business Media, LLC. ISBN: 978-0-387-09505-9.

Dotta, S., Jorge, E., Braga, J.,; Pimentel, E. (2012) "Relato de Experiência: Processo de Elaboração de um Curso à Distância Utilizando a Metodologia Intera".ESUD 2012 - IX Congresso Brasileiro de Ensino Superior a Distância. Recife - PE.

Siller, F.; Braga, J. C. (2013). Desenvolvimento de um jogo para prática do Scrum. Trabalho de Graduação. Universidade Federal do ABC.

CETIC - Pesquisa TIC Educação 2012 - Pesquisa sobre o uso das TIC nas escolas brasileira http://www.cetic.br/educacao/index.htm.

Filatro A. Design instrucional contextualizado. São Paulo: Senac, 2004.

RIVED (2008), "Conheça o RIVED", Disponível em: http://www.rived.mec.gov.br/conheca_rived.php.

Kruchten, P. (2003) "Rational Unified Process Made Easy-A Practitioner's Guide to the RUP", with Per Kroll, Addison-Wesley. 2003.

Pessoa, M. C e Benitti F. B. V. (2008) "Proposta de um processo para produção de objetos de aprendizagem". Porto Alegre. Hifen. 6, 7.

PMBOK - Project MANAGEMENT Institute (Pmi), Standards Committee, 2008, "A Guide to the Project Management Body of knowledge (PMBOK)" Quarta edição

SWEBOK, Guide to Software Engineering Body of Knowledge (SWEBOK). IEEE Computer Society. (2004). Disponível em: http://www.swebok.org/. 